\title{
Análisis de propiedades psicométricas del Índice de Bienestar Personal en una muestra de adolescentes chilenos*
}

\section{Psychometric Properties Analysis of the Personal Well-Being Index in a Sample of Chilean Adolescents}

\author{
Jaime Alfaro Inzunza** \\ Universidad del Desarrollo, Chile \\ Boris Valdenegro Egozcue \\ Universidad de Chile, Chile \\ Denise Oyarzún Gómez \\ Pontificia Universidad Católica \\ de Valparaíso, Chile \\ Recibido: 2 de abril de 2012 \\ Revisado: 22 de julio de 2012 \\ Aceptado: 14 de septiembre de 2012
}

\section{Resumen}

En el artículo se reportan las propiedades psicométricas de la versión chilena del Índice de Bienestar Personal (Cummins et ál., 2003) en tres modalidades: PWI 7, PWI + espiritualidad y PWI + religiosidad, en una muestra de 1621 adolescentes chilenos de ambos sexos, entre 14 y 18 años, formados en establecimientos educativos municipales y particulares subvencionados de Gran Valparaíso. Los análisis de fiabilidad (alfa de Cronbach) indican niveles adecuados de consistencia interna para las tres modalidades. El análisis de componentes principales señala un factor único en todas las modalidades y permite observar valores diferenciales de varianza explicada. El análisis de regresión arroja valores $\mathrm{R}^{2}$ similares para las distintas versiones, excluyéndose en todos los modelos los ámbitos "con tu salud" y "con los grupos".

Palabras clave: propiedades psicométricas, bienestar personal, espiritualidad, religión, adolescencia.

Artículo de investigación. En este trabajo, salvo algunas variables demográficas agregadas, el cuestionario fue el mismo proporcionado por el Equip de Recerca sobre Infants, Adolescents, Drets dels Infants i la seva Qualitat de Vida (ERIDIQV).

** Correspondencia: Jaime Alfaro Inzunza, Facultad de Psicología, Universidad del Desarrollo (UDD), Santiago, Chile; Boris Valdenegro Egozcue, Programa de Doctorado en Psicología de la Universidad de Chile (UCh), Santiago de Chile; Denise Oyarzún Gómez, Programa de Doctorado en Psicología, Pontificia Universidad Católica de Valparaíso, Avenida El Bosque \#1290, Viña del Mar, Chile. La correspondencia relativa a este artículo debe ser dirigida a Jaime Alfaro Inzunza, Facultad de Psicología de la Universidad del Desarrolllo, Avenida La Plaza 700 Las Condes, Santiago, Chile. Correo electrónico: jalfaro@udd.cl 


\section{Abstract}

We report the psychometric properties of the Chilean version of the Personal Well-being Index (Cummins et ál., 2003) in its three forms: PWI7, PWI + spirituality, PWI + religiosity, in a sample of 1621 Chilean adolescents of both sexes between 14 and 18 years, of municipal schools and private subsidized the Greater Valparaíso. The reliability analysis (Cronbach's alpha) indicates adequate levels of internal consistency for the three versions. The main analysis component indicates a single factor in all versions, with values of explained variance differentials. The regression analysis yields similar $\mathrm{R}^{2}$ values for the different versions, all models excluded areas "To your health" and "With groups".

Keywords: psychometric properties, personal well-being, spirituality, religion, adolescents.

\section{Bienestar subjetivo: antecedentes generales}

La noción de bienestar subjetivo está constituida por tres dimensiones interrelacionadas: los afectos positivos, los afectos negativos y la satisfacción con la vida (Diener y Suh, 1997; Pavot y Diener, 1993). Otros autores consideran también el componente felicidad (Casas, 2010b). Los afectos refieren a las emociones, mientras que la satisfacción con la vida se refiere a un juicio cognitivo global que hacen las personas respecto a la satisfacción con sus vidas (Diener y Suh, 1997).

La satisfacción vital es entendida como una evaluación global de la persona sobre la calidad de su vida ante sus circunstancias (Seligson, Huebner y Valois, 2003), lo que da cuenta del grado en que evalúa positivamente su vida como globalidad o totalidad (Veenhoven, 1994), o en relación con ámbitos específicos tales como la familia, los amigos o la escuela (Huebner, 2004; Seligson, Huebner y Valois, 2005). Ello es relativo a un estado presente, aunque no momentáneo (Veenhoven, 1994).

Aunque este constructo considera tanto una apreciación cognitiva a la vez que afectiva (Veenhoven, 1994) y puede ser indirectamente influido por los afectos o estados emocionales transitorios (Huebner, 2004; Seligson et ál., 2005), el componente evaluativo es dominante (Lau, Cummins y McPherson, 2005; Seligson et ál., 2005), de tal forma que la noción satisfacción con la vida expresa una respuesta de carácter básica- mente cognitivo (Pavot y Diener, 1993; Huebner, 2004; Pavot y Diener, 1993).

Según Veenhoven (1994), los datos de satisfacción con la vida, en cuanto permiten calcular la calidad de vida "realizada" en un país o categoría social, son útiles en diversos aspectos: en la validación empírica de ideas acerca de las condiciones necesarias para el vivir bien; acerca de qué es una buena sociedad; y para evaluar efectos en la calidad de vida de las políticas aplicadas, lo que es de gran importancia para trazar estrategias de desarrollo social (Veenhoven, 2002).

Seligson et ál. (2003) establecen cuatro importantes ventajas de esta noción respecto a la promoción de la salud: permite integrar las perspectivas psicológicas y sociológicas con las perspectivas médicas y de rehabilitación; permite destacar y acceder a los múltiples factores determinantes de la salud, incluyendo dimensiones personales, comunitarias y los factores determinantes estructurales; al identificar estos factores determinantes de la salud, permite la activación de condiciones necesarias para comportamientos saludables, y con ello contribuye a la implementación de acciones de promoción y rehabilitación de la salud; finalmente, permite desarrollar un instrumental sensible para evaluar la capacidad de las intervenciones, a fin de incidir en los problemas de salud/enfermedad.

Así, el estudio y desarrollo de medidas de satisfacción con la vida permite determinar riesgos en dimensiones tanto psicológicas como sociales, lo 
cual representa una valiosa capacidad predictiva respecto a estados depresivos y a la capacidad de manejo de eventos vitales estresantes y sus efectos en problemas de comportamiento. De este modo, proporciona una importante información para la prevención, detección precoz e intervención en diversas poblaciones en condiciones de riesgo (Seligson et ál., 2005).

Existe un acuerdo entre los investigadores de que la disponibilidad de estudios sobre el bienestar de niños, niñas y adolescentes dista mucho de alcanzar la cantidad de resultados que ofrece la investigación con adultos (Casas, 2010b; Casas et ál., 2011b; Huebner, 2004; Huebner y Gilman, 2002; Gademann, Schonert-Reichl y Zumbo, 2010; Seligson et ál., 2003, 2005; Tomyn y Cummins, 2011) tanto cuantitativa como cualitativamente (Casas, 2010b).

Así entendido, el estudio del bienestar subjetivo en la infancia y adolescencia es un campo en inicio con muchos desafíos aún, especialmente en población no clínica (Casas, 2010). Según Pollard y Lee (2003), los trabajos producidos referirían de manera principal a población en condiciones médicas crónicas, o se orientarían a la identificación de indicadores "negativos". En este sentido, existía una necesidad de investigación que se extiende más allá del estudio de los trastornos, déficits y discapacidades en los niños (Gademann et ál., 2010).

El avance en la medición del bienestar subjetivo en la infancia y adolescencia permitiría aumentar nuestra comprensión de los correlatos de este, medir su estado y guiar las acciones tendentes a su elevación y mejora, lo que sería complementado con una aproximación centrada en la evaluación de las conductas problemáticas o dimensiones patológicas de estos (Gademann et ál., 2010). Esto, además, permitiría conocer sistemáticamente los factores relacionados con las diferencias individuales en la percepción del bienestar y sus secuelas (Huebner y Gilman, 2002; Seligson et ál., 2003, 2005), así como la identificación de subgrupos de riesgo en función de garantizar la adecuada prestación de apoyo y recursos a los que lo necesitan (Tomyn y Cummins, 2011).
Desde esta perspectiva, una de las principales deficiencias de la matriz actual de medidas de bienestar disponibles para los niños, niñas y adolescentes se relaciona con la necesidad de avanzar en el desarrollo de instrumental de medición breve y multidimensional de la satisfacción con la vida, respecto a su uso en estudios a gran escala (Huebner y Gilman, 2002; Seligson et ál., 2005). Lo anterior se hace más relevante si se tiene en cuenta que importantes estudios nacionales y transnacionales en calidad de vida de los adultos se han emprendido gracias a la disponibilidad de este tipo de instrumental, con un rendimiento considerable tanto teórico como de generación de información práctica (Seligson et ál., 2003).

Así, el avance en el estudio de satisfacción de vida requiere el desarrollo de nuevas medidas, en particular, medidas comparativas nacionales y transnacionales, como también de grupos o entornos culturales diversos (Casas, 2010b; Casas et ál., 2011b; Siyez y Kaya, 2008). Existen hasta ahora escasas publicaciones con prueba empírica de los resultados obtenidos con diferentes instrumentos aplicados a infancia y adolescencia en diferentes países o contextos culturales. La mayoría de estos estudios han sido realizados en el mundo anglosajón, con algunas excepciones (Casas et ál., 2011b).

Ello es particularmente importante si consideramos, como señala Casas (2010b), que no se puede dar por sentado que los resultados con muestras de adultos son extrapolables a poblaciones jóvenes del mismo contexto sociocultural. Por el contrario, los pocos datos disponibles tienden a sugerirnos que durante las etapas más jóvenes de la vida, el funcionamiento del bienestar tiene su propia idiosincrasia evolutiva a lo largo de los años (Casas, 2010b).

Una consideración general que se debe tener en cuenta es que el ámbito de investigación en bienestar subjetivo y sus nociones asociadas en términos generales, incluyendo la población infantil, adolescente y de adultos, no se ha producido de manera significativa en países en vías de desarrollo, tal cual reportan Lau, Cummins y McPherson (2005); Lim y Sleigh (2010); Siyez y Kaya (2008); 
Tiliouine, Cummins y Davern (2006); Webb (2009); Yiengprugsawan, Seubsman y Khamman (2010).

Conocemos reportes con base en el Índice de Bienestar Personal (PWI) aplicados solo en países en vía de desarrollo, tales como Tailandia (Yiengprugsawan et ál., 2010), Argelia (Tiliouine et ál., 2006), o con la Escala Breve Multidimensional de Satisfacción con la Vida en Estudiantes (BMSLSS) en Turquía (Siyez y Kaya, 2008).

Esta necesidad investigativa es particularmente relevante en América Latina, región en donde sabemos muy poco sobre la calidad de vida de los niños y adolescentes, en particular sobre su calidad de vida en dimensiones subjetivas, según sus diversos contextos y la diversidad de situaciones colectivas de la región (Aguado, 2009).

Ello contrasta con la necesidad de avanzar en el sentido que indica Ben-Arieh (2008), quien en una revisión reciente de la historia de los indicadores sociales de la infancia señala que la necesidad de desarrollar indicadores positivos y subjetivos para población infantil y adolescente se relaciona con las tendencias que se observan en este campo. Según plantea, en un primer periodo, los indicadores sociales para esta población solo incluían "indicadores de supervivencia", tales como tasas de mortalidad y tasas de enfermedades y problemas sociales que afectan a la infancia como, por ejemplo, desescolarización, analfabetismo, fracaso escolar, etc. En cambio, en los últimos treinta años habrían emergido perspectivas subjetivas que hacen necesario considerar explicaciones del mundo desde la visión del niño, centrándose en la infancia como unidad de observación, en vez de la familia, la escuela o la comunidad.

En igual sentido apuntan Bradshow y Lau (2010) y Casas (2010a), señalando que en la actualidad se hace necesario incluir las nociones de calidad de vida (no solo la satisfacción de necesidades) y bienestar subjetivo como elementos relevantes en el desarrollo de indicadores sociales en la infancia, considerando a los niños como sujetos de derechos.

\section{Índice de Bienestar Personal (PWI)}

El Índice de Bienestar Personal es una escala diseñada por Cummins et ál. (2003) y recientemente modificada por el International Wellbeing Group (2006). El PWI contiene ocho reactivos referidos a la satisfacción con diversos ámbitos de calidad de vida: el nivel de vida, la salud, el logro en la vida, las relaciones, la seguridad, los vínculos con la comunidad, la seguridad en el futuro y la espiritualidad o religión. Estos ocho ámbitos son teóricamente integrados, en tanto son la representación global de la satisfacción con la vida (International Wellbeing Group, 2006).

Conceptualmente, el PWI está centrado solo en el componente satisfacción con la vida del bienestar subjetivo (Cummins et ál., 2003; International Wellbeing Group, 2006) y ha excluido el componente afecto/felicidad que tenía la escala original (ComQol) desde donde fue derivado (Lau et ál., 2005, 2008; Smyth, Nielsen y Zhai, 2010).

Muestra un buen rendimiento de validez, fiabilidad y sensibilidad en poblaciones de adultos de todas las edades y en variados países (International Wellbeing Group, 2006; Tiliouine et ál., 2006). La combinación de la variación única y compartida por los ocho ámbitos típicamente explica alrededor del $30-60 \%$ de la variación en la satisfacción con la vida como un todo. La buena homogeneidad interna se demuestra con valores alfa de Cronbach de entre 0,75 y 0,85 (International Wellbeing Group, 2006; Lau et ál., 2008).

El PWI está siendo utilizado por más de cien investigadores en cincuenta países y ha sido traducida a varios idiomas, tales como el chino, alemán, japonés, ruso, español, entre otros (International Wellbeing Group, 2006). No obstante, presenta interrogantes sobre la aplicación de sus hallazgos y sobre sus propiedades en países en vía de desarrollo (Tiliouine et ál., 2006).

A pesar de que el PWI fue diseñado para población adulta (Lau et ál., 2005), la construcción de sus ítems está planteada de forma suficientemente 
amplia para ser aplicada a todo tipo de poblaciones. Complementariamente, permite aplicaciones breves de nivel masivo (Lau et ál., 2008).

Uno de los problemas no resueltos de la investigación con este instrumento en población adolescente es que la varianza explicada es generalmente mucho menor que con muestras de adultos del mismo contexto sociocultural, lo que sugiere que faltan otros ámbitos para los adolescentes en la forma de esta escala (Casas et ál., 2011) y/o que los ámbitos presentes no son los más relacionados con la satisfacción personal.

Asimismo, en los últimos años ha surgido un debate respecto a la satisfacción con la espiritualidad o religión (Casas, González, Figuer y Malo, 2009; Casas, 2010b; Casas et ál., 2011b). Este tema ha demostrado ser problemático cuando se comparan diferentes contextos socioculturales $y$, en particular, cuando se utiliza con muestras de adolescentes, ya que parece que influyen diferentes significados en sus resultados (Casas et ál., 2011b).

Según reporta el International Wellbeing Group (2006) en población australiana, el ámbito espiritual o religión no hace una contribución diferenciada, pero sí ha demostrado hacerlo en Colombia. A su vez, estudios realizados en España (Cataluña) (Casas et ál., 2009) reportan que la mayoría de los jóvenes considera que la religión tradicional no es muy importante para su bienestar, mientras que la importancia dada a la espiritualidad es claramente superior. Al comparar las respuestas de los adolescentes con los de sus padres, observan que la contribución a la satisfacción de todos los ámbitos fue superior respecto a la de sus padres, a excepción de la satisfacción con la religión o espiritualidad.

Según se concluye en estos estudios (Casas et ál., 2009) quedan planteadas dudas respecto a si estos resultados, por un lado, se pueden entender como parte de un proceso de secularización contextual, estando los jóvenes menos satisfechos con este ámbito, o si bien, por otro lado, se trata de un problema de comprensión y de atribución de significado a esta dimensión debido a su alto nivel de la abstracción.
Sin embargo, al estudiar las respuestas separando un ítem referido a satisfacción con la espiritualidad y otro a religión, los resultados muestran que, siendo la contribución a la satisfacción global baja en ambos casos, en comparación con los demás ámbitos, el subámbito más bajo es la religión y no la espiritualidad (Casas et ál., 2009). Lo anterior es válido tanto para los jóvenes adolescentes como para jóvenes universitarios de mayor edad, mientras que, por el contrario, la satisfacción con la espiritualidad aumenta ligeramente para los grupos de mayor edad, tanto en términos absolutos como respecto a su influencia relativa en la satisfacción global (Casas et ál., 2009).

Se resalta así la necesidad de seguir explorando el funcionamiento de la escala de PWI en adolescentes con satisfacción con la religión y la espiritualidad como ámbitos juntos y por separado, y su relación con edad y en diferentes culturas, con el fin de comprender mejor su contribución al bienestar en estas poblaciones específicas.

Desde este planteamiento, se considera relevante el análisis del funcionamiento diferencial de la escala en la realidad chilena, según las particulares distinciones propuestas. Para tal efecto se constituyeron tres versiones de la escala para su análisis, las cuales se diferenciaron en relación con la inclusión o exclusión de los ámbitos de espiritualidad o religiosidad:

- Versión PWI 7: "con tu salud", "con tu nivel de vida", "con las cosas que has conseguido en la vida", "con lo seguro o segura que te sientes", "con los grupos de gente de los que formas parte", "con tu seguridad por tu futuro", "con tus relaciones con otras personas".

- Versión PWI 7 + espiritualidad: a los anteriores reactivos se incluye "con tu espiritualidad".

- Versión PWI 7 + religiosidad: a los reactivos del PWI 7 se incluye "con tu religión".

Este estudio analiza las propiedades psicométricas del Índice de Bienestar Personal de Cummins, traducido y adaptado del catalán a la versión chilena, en una muestra de 1621 adolescentes chilenos 
de ambos sexos, pertenecientes a la región de Valparaíso, con edades entre los 14 y 18 años. Lo anterior se desarrolla a través de un análisis comparativo de tres modalidades del PWI: PWI 7, PWI 7 + espiritualidad y PWI 7 + religiosidad, considerando el análisis de la fiabilidad interna (alfa de Cronbach) y la validez de constructo (análisis de componentes principales). Se realiza conjuntamente el análisis del aporte diferencial de cada ámbito de satisfacción a la noción de satisfacción global: regresión lineal múltiple sobre el ítem único de satisfacción global (OLS).

\section{Método}

\section{Muestra}

Los participantes que componen la muestra son 1621 estudiantes de secundaria, pertenecientes a establecimientos públicos de la región de Valparaíso, Chile. Las edades fluctuaron entre los 14 y los 18 años, con una media de 15,9 años y desviación típica de 1,2 años. Las frecuencias relativas según tramos de edad fueron las siguientes: para los 14 años, del 13,6\%; para los 15 años, del 24,4\%; para los 16 años, del 27,0\%; para los 17 años, del 24,4\%; y para los 18 años, del 10,7\%. La división por sexo fue de $47 \%$ para los hombres y de $53 \%$ para las mujeres.

\section{Instrumentos}

\section{Índice de Bienestar Personal}

Esta escala se orienta a la medición del bienestar subjetivo en diversos ámbitos de satisfacción: estándar de vida, salud, logros vitales, relaciones personales, seguridad personal, seguridad futura, relaciones con los grupos y espiritualidad o religiosidad. Estos ámbitos de satisfacción se presentan relacionados, según los autores, con el bienestar global de las personas, traduciéndose cada uno de ellos en un ítem específico.

Los reportes de fiabilidad de la escala (alfa de Cronbach) reseñados por el autor entregan valores entre 0,70 y 0,85 , siendo las correlaciones totales del ítem de al menos 0,50 (International Wellbeing Group, 2006).

En noviembre de 2006 es incluido por los autores el ámbito de religiosidad/espiritualidad (International Wellbeing Group, 2006). Dado los antecedentes presentados sobre los resultados del octavo ítem, en la aplicación del presente estudio se consideraron dos versiones distintas de este: una referida exclusivamente a espiritualidad y otra a religiosidad. De este modo, si bien para fines de la aplicación de la muestra se trata de una escala de nueve reactivos, para efectos del análisis diferencial se establecen la existencia de tres versiones: PWI 7, PWI 7 + espiritualidad y PWI 7 + religiosidad.

En la presentación final de la escala para Chile, los reactivos correspondientes a los distintos ámbitos de satisfacción con la vida se plantean en evaluación ante la siguiente proposición: "Actualmente, ¿hasta qué punto te encuentras satisfecho o satisfecha con cada una de estas cosas de tu vida?". Se incluyeron de modo común los nueve reactivos (correspondientes a distintos ámbitos de satisfacción) en su aplicación a la muestra en estudio, enunciados de la siguiente manera: "con tu salud", "con tu nivel de vida", "con las cosas que has conseguido en la vida", "con lo seguro o segura que te sientes", "con los grupos de gente de los que formas parte", "con tu seguridad por tu futuro", "con tus relaciones con otras personas", "con tu espiritualidad" y "con tu religión".

La duración de la aplicación fue de 45 a 60 minutos.

\section{Ítem único de satisfacción global con la vida}

Este ítem no hace parte del PWI, pero se emplea en diversas investigaciones para analizar la validez del constructo de la escala (International Wellbeing Group, 2006), así como para determinar la contribución específica de cada ítem a la varianza total. Consiste en una pregunta formulada en los siguientes términos: "Pensando en tu vida actual, ¿cuán satisfecho(a) te encuentras en tu vida considerada globalmente?". 


\section{Procedimiento}

El cuestionario fue adaptado para el castellano de uso en Chile desde su versión catalana. Ello se efectuó por primera vez para el presente estudio, según el método de traducción simple y con la aplicación de un estudio piloto.

La aplicación final fue desarrollada en la modalidad de autoaplicación durante horarios de clase, tiempo facilitado por los profesores de manera previa al contacto con las autoridades de los establecimientos educacionales. Siempre estuvo presente un responsable del equipo de investigación durante la aplicación, para resolver las dudas de los encuestados. También se cumplieron los protocolos éticos de investigación que orientan la investigación científica (Conicyt/Fondecyt, 2008), con especial énfasis en la confidencialidad de la información producida.

La escala se aplicó a través de la modalidad de autoadministración durante el periodo escolar 2010 y en el horario regular de clases. Con posterioridad a su tabulación en una matriz de datos, los resultados obtenidos se han orientado a determinar algunas propiedades psicométricas de relevancia del instrumento, en orden a determinar los coeficientes de confiabilidad de la aplicación, a explorar la correspondencia de su estructura factorial con la propuesta por el autor y a determinar el aporte específico de los nueve reactivos a la varianza, la cual es explicada a través de la aplicación de un procedimiento de regresión lineal múltiple sobre el ítem único de satisfacción global con la vida, según la propuesta de Cummins (2006).

\section{Resultados}

\section{Resultados estadísticos descriptivos y confiabilidad interna de la escala}

En la tabla 1 se detallan los resultados estadísticos de tendencia central relevantes (media y desviación estándar) de los distintos reactivos.
Tabla 1.

Estadísticos descriptivos

\begin{tabular}{lcc}
\hline \multicolumn{1}{c}{ Ámbitos } & Media & $\begin{array}{c}\text { Desviación } \\
\text { típica }\end{array}$ \\
\hline Salud & 7,65 & 2,47 \\
Nivel de vida & 7,99 & 2,09 \\
Logros de vida & 7,72 & 2,19 \\
Seguridad sentida & 7,27 & 2,49 \\
Grupos de los que & 8,42 & 2,07 \\
forman parte & 7,11 & 2,56 \\
Seguridad a futuro & 8,06 & 2,13 \\
Relación con las & & \\
Personas & & 2,65 \\
Espiritualidad & 7,29 & 3,39 \\
Religión & 6,69 & \\
\hline
\end{tabular}

Fuente: autores.

El análisis de confiabilidad de las distintas versiones del instrumento (con o sin inclusión de los ámbitos religiosidad y espiritualidad) aparece contenido en la tabla 2. Como se aprecia en esta, los valores oscilan entre 0,806 y 0,74 . No obstante, se aprecia una baja en la confiabilidad desde la inclusión del ítem referido al ámbito de la religiosidad en tanto ámbito de satisfacción (elevándose a 0,805 al eliminarse), lo que se reafirma en la correlación ítem-test (corregida) respecto a dicho ámbito $(0,251)$, que es ostensiblemente menor que el resto de los ámbitos. De modo complementario, la inclusión del ámbito de satisfacción relacionado con la espiritualidad en la correspondiente versión del PWI hace aumentar ligeramente la confiabilidad (el alfa disminuye si esta se elimina). 
Tabla 2.

Resultados estadísticos de fiabilidad y correlación item-total (corregida)

\begin{tabular}{lccc}
\hline Ámbitos & $\begin{array}{c}\text { Ítem del } \\
\text { test PWI 7 }\end{array}$ & $\begin{array}{c}\text { Ítem del } \\
\text { test PWI 7 } \\
\text { espiritua- } \\
\text { lidad }\end{array}$ & $\begin{array}{c}\text { Ítem del } \\
\text { test PWI 7 } \\
\text { +religiosi- } \\
\text { dad }\end{array}$ \\
\hline Salud & 0,475 & 0,469 & 0,455 \\
$\begin{array}{l}\text { Nivel de } \\
\text { vida }\end{array}$ & 0,567 & 0,565 & 0,554 \\
$\begin{array}{l}\text { Logros de } \\
\text { vida }\end{array}$ & 0,604 & 0,607 & 0,586 \\
$\begin{array}{l}\text { Seguridad } \\
\text { sentida }\end{array}$ & 0,615 & 0,614 & 0,588 \\
$\begin{array}{l}\text { Grupos } \\
\text { de los } \\
\text { que for- } \\
\text { man parte }\end{array}$ & 0,476 & 0,476 & 0,463 \\
$\begin{array}{l}\text { Seguridad } \\
\text { a futuro }\end{array}$ & 0,513 & 0,515 & 0,513 \\
$\begin{array}{l}\text { Relación } \\
\text { con las } \\
\text { personas } \\
\begin{array}{l}\text { Espirituali- } \\
\text { dad }\end{array}\end{array}$ & 0,537 & 0,546 & 0,531 \\
$\begin{array}{l}\text { Religión } \\
\text { Versiones }\end{array}$ & PWI 7 & PWI 7 + esp. & PWI 7 + rel. \\
\hline PWI & -- & 0,399 & - \\
\hline
\end{tabular}

\begin{tabular}{lccc}
\hline Ámbitos & $\begin{array}{c}\text { Ítem del } \\
\text { test PWI 7 }\end{array}$ & $\begin{array}{c}\text { Ítem del } \\
\text { test PWI 7 } \\
\text { +espiritua- } \\
\text { lidad }\end{array}$ & $\begin{array}{c}\text { test PWI 7 } \\
\text { religiosi- } \\
\text { dad }\end{array}$ \\
\hline $\begin{array}{l}\text { Alfa de } \\
\text { Cronbach }\end{array}$ & 0,805 & 0,806 & 0,740 \\
\hline
\end{tabular}

Fuente: autores.

Asimismo, se observan valores en las correlaciones ítem-total (corregida) de los ámbitos religiosidad y espiritualidad, comparativamente menores respecto a los ámbitos originales (PWI 7). Del mismo modo, se aprecia una relativa estabilidad en las correlaciones de los restantes ámbitos entre las distintas versiones del PWI. Los rangos de las correlaciones ítem-total (corregida) de los distintos ámbitos (exceptuando religiosidad y espiritualidad) van entre $r(0,46)$ y $r(0,62)$, con $p \leq 0,01$.

\section{Correlación entre ámbitos de bienestar}

De acuerdo con los resultados en la tabla 3 , se aprecia que los rangos de correlación entre los ámbitos van entre $r(0,15), p \leq 0,01$ y $r(0,47), p \leq$ 0,01 . Los ámbitos con mayores niveles de correlación son logros de la vida y nivel de vida, $r(0,47)$, $p \leq 0,01$, seguido de salud y nivel de vida, $r(0,46)$, $p \leq 0,01$; salud y religión, $r(0,15), p \leq 0,01$, son los que presentan las correlaciones más bajas.

Tabla 3.

Correlaciones entre ámbitos

\begin{tabular}{|c|c|c|c|c|c|c|c|c|c|}
\hline Ámbitos & Salud & $\begin{array}{l}\text { Nivel de } \\
\text { vida }\end{array}$ & $\begin{array}{l}\text { Logros } \\
\text { de vida }\end{array}$ & $\begin{array}{l}\text { Seguridad } \\
\text { sentida }\end{array}$ & Grupos & $\begin{array}{l}\text { Seguridad } \\
\text { a futuro }\end{array}$ & $\begin{array}{c}\text { Relación } \\
\text { con las } \\
\text { personas }\end{array}$ & Espiritualidad & Religión \\
\hline Salud & 1,000 & & & & & & & & \\
\hline $\begin{array}{l}\text { Nivel de } \\
\text { vida }\end{array}$ & 0,457 & 1,000 & & & & & & & \\
\hline $\begin{array}{l}\text { Logros de } \\
\text { vida }\end{array}$ & 0,326 & 0,465 & 1,000 & & & & & & \\
\hline $\begin{array}{l}\text { Seguridad } \\
\text { sentida }\end{array}$ & 0,303 & 0,369 & 0,491 & 1,000 & & & & & \\
\hline $\begin{array}{l}\text { Grupos de } \\
\text { los que } \\
\text { formas } \\
\text { parte }\end{array}$ & 0,232 & 0,310 & 0,328 & 0,360 & 1,000 & & & & \\
\hline
\end{tabular}




\begin{tabular}{|c|c|c|c|c|c|c|c|c|c|}
\hline Ámbitos & Salud & $\begin{array}{l}\text { Nivel de } \\
\text { vida }\end{array}$ & $\begin{array}{l}\text { Logros } \\
\text { de vida }\end{array}$ & $\begin{array}{c}\text { Seguridad } \\
\text { sentida }\end{array}$ & Grupos & $\begin{array}{l}\text { Seguridad } \\
\text { a futuro }\end{array}$ & $\begin{array}{c}\text { Relación } \\
\text { con las } \\
\text { personas }\end{array}$ & Espiritualidad & Religión \\
\hline $\begin{array}{l}\text { Seguridad } \\
\text { a futuro }\end{array}$ & 0,245 & 0,306 & 0,421 & 0,443 & 0,270 & 1,000 & & & \\
\hline $\begin{array}{l}\text { Relación } \\
\text { con las } \\
\text { personas }\end{array}$ & 0,269 & 0,315 & 0,384 & 0,405 & 0,433 & 0,348 & 1,000 & & \\
\hline $\begin{array}{l}\text { Espiritua- } \\
\text { lidad }\end{array}$ & 0,194 & 0,284 & 0,319 & 0,285 & 0,227 & 0,245 & 0,299 & 1,000 & \\
\hline Religión & 0,146 & 0,209 & 0,204 & 0,194 & 0,178 & 0,201 & 0,223 & 0,465 & 1,000 \\
\hline
\end{tabular}

Nota: Todas las correlaciones son significativas (a dos colas) con $p \leq 0,01$.

Fuente: autores.

\section{Validez de constructo: análisis de componentes principales}

Para abordar la estructura interna del instrumento se realizó un análisis de componentes principales, teniendo en cuenta cada una de las versiones del instrumento. Los resultados referidos a la adecuación de este procedimiento de análisis se encuentran contenidos en la tabla 4 y dan cuenta de la viabilidad de realizar el análisis factorial (exploratorio).

Tabla 4.

Parámetros de plausibilidad

\begin{tabular}{lcc}
\hline \multicolumn{1}{c}{ Escala } & $\begin{array}{c}\text { Esfericidad } \\
\text { de Bartlett }\end{array}$ & $\begin{array}{c}\text { Adecuación } \\
\text { muestral (Kaiser, } \\
\text { Meyer, Olkin) }\end{array}$ \\
\hline PWI 7 & $\begin{array}{l}3003,716, \\
\mathrm{p} \leq 0,0001\end{array}$ & 0,844 \\
PWI 7 + & 3117,565 & 0,850 \\
religiosidad & $\mathrm{p} \leq 0,0001$ & \\
$\begin{array}{l}\text { PWI7 + } \\
\text { espiritualidad }\end{array}$ & $\begin{array}{c}3294,242 \\
\mathrm{p} \leq 0,0001\end{array}$ & 0,862 \\
\hline
\end{tabular}

Fuente: autores.

De acuerdo con el análisis de componentes principales, emerge una estructura monofactorial del PWI en todas las versiones del test. Se aprecia asimismo la saturación de los siete ámbitos clásicos (excluyendo religiosidad y espiritualidad) en- tre los rangos 0,620 y 0,748 , según se observa en la siguiente tabla.

Tabla 5.

Cargas factoriales de los ítems y porcentajes de varianza explicada

\begin{tabular}{|c|c|c|c|}
\hline Ámbitos & $\begin{array}{c}\text { PWI } 7 \\
\text { Comp. } \\
1\end{array}$ & $\begin{array}{l}\text { PWI7 + } \\
\text { religiosidad } \\
\text { Comp. } \\
1\end{array}$ & $\begin{array}{c}\text { PWI7 + } \\
\text { espiritualidad } \\
\text { Comp. } \\
1\end{array}$ \\
\hline $\begin{array}{l}\text { Con lo seguro o } \\
\text { segura que te } \\
\text { sientes }\end{array}$ & 0,748 & 0,742 & 0,742 \\
\hline $\begin{array}{l}\text { Con cosas que has } \\
\text { conseguido en la } \\
\text { vida }\end{array}$ & 0,739 & 0,735 & 0,735 \\
\hline $\begin{array}{l}\text { Con tu nivel de } \\
\text { vida }\end{array}$ & 0,702 & 0,699 & 0,694 \\
\hline $\begin{array}{l}\text { Con tus relacio- } \\
\text { nes con las otras } \\
\text { personas }\end{array}$ & 0,677 & 0,677 & 0,676 \\
\hline $\begin{array}{l}\text { Con la seguridad } \\
\text { por tu futuro }\end{array}$ & 0,656 & 0,658 & 0,650 \\
\hline $\begin{array}{l}\text { Con los grupos de } \\
\text { los que formas } \\
\text { parte }\end{array}$ & 0,620 & 0,616 & 0,612 \\
\hline $\begin{array}{l}\text { Con tu salud, con } \\
\text { tu religión y con } \\
\text { tu espiritualidad }\end{array}$ & 0,620 & $\begin{array}{l}0,613 \\
0,347\end{array}$ & $\begin{array}{l}0,608 \\
0,524\end{array}$ \\
\hline $\begin{array}{l}\text { \% de varianza } \\
\text { explicada }\end{array}$ & 46,539 & 41,816 & 43,378 \\
\hline Autovalor & 3,258 & 3,345 & 3,470 \\
\hline
\end{tabular}

Fuente: autores. 
Llama la atención la notoria diferencia de la magnitud en la carga factorial de los ámbitos con tu religión $(0,347)$ y en menor medida con tu espiritualidad $(0,524)$, para las respectivas versiones del instrumento, que, sin embargo, siguen siendo significativas.

La varianza explicada desde el análisis factorial evidencia que las versiones del PWI con inclusión de los ámbitos espiritualidad y religiosidad presentan menor varianza explicada que la versión del PWI 7. Complementariamente, se aprecia que la mayor varianza explicada se encuentra en el PWI 7, seguido por PWI + espiritualidad.

\section{Contribuciones específicas de los ámbitos a la dimensión "satisfacción con tu vida considerada globalmente"}

Para comprobar los aportes diferenciales de los ámbitos del PWI en sus distintas versiones, se procedió a efectuar una regresión lineal con el factor "satisfacción con tu vida considerada globalmente" (OLS).

Los valores de $R^{2}$ (ajustados según grados de libertad) aparecen en la tabla 6, junto con los ítems que se excluyen del modelo, para cada versión de la escala PWI, oscilando entre $0,403[F(5,1615)$ $=219,618, p \leq 0,01]$ y $0,426[\mathrm{~F}(6,1614)=201,586$, $p \leq 0,01]$.

Tabla 6.

Resultados $R^{2}$

\begin{tabular}{|c|c|c|c|}
\hline Escala & $\begin{array}{c}\mathbf{R}^{2} \\
\text { ajustado }\end{array}$ & $\begin{array}{c}\text { Ítems } \\
\text { excluidos }\end{array}$ & $t$ \\
\hline PWI 7 & 0,403 & $\begin{array}{l}\text { "Con tu salud", } \\
\text { "con los grupos } \\
\text { de los que formas } \\
\text { parte" }\end{array}$ & $\begin{array}{l}1,593 \\
1,431\end{array}$ \\
\hline $\begin{array}{l}\mathrm{PWI}+ \\
\text { religiosidad }\end{array}$ & 0,426 & $\begin{array}{l}\text { "Con tu salud", } \\
\text { "con los grupos } \\
\text { de los que formas } \\
\text { parte" }\end{array}$ & $\begin{array}{l}1,561 \\
1,277\end{array}$ \\
\hline
\end{tabular}

\begin{tabular}{lclc}
\hline \multicolumn{1}{c}{ Escala } & $\begin{array}{c}\mathbf{R}^{2} \\
\text { ajustado }\end{array}$ & \multicolumn{1}{c}{$\begin{array}{c}\text { Ítems } \\
\text { excluidos }\end{array}$} & $\mathbf{t}$ \\
\hline $\begin{array}{l}\text { PWI + } \\
\text { espirituali- } \\
\text { dad }\end{array}$ & 0,414 & $\begin{array}{l}\text { “Con tu salud", } \\
\text { "con los grupos } \\
\text { de los que formas } \\
\text { parte”. }\end{array}$ & 1,414 \\
\hline
\end{tabular}

Fuente: autores.

Para la versión PWI 7, en el análisis de regresión, la varianza explicada llega al 40,3\% [F $(5,1615)$ $=219,618, p \leq 0,01]$ y conserva una correlación parcial sobre 0,1 para todos los ítems (criterio de decisión para cada modelo escogido). Se excluyen con tu salud $(B=0,036, p=0,112)$ y con los grupos de los que formas parte $(B=0,032, p=0,152)$, como parte de los ítems integrados al modelo, por no alcanzar valores de significancia.

Respecto de la versión PWI + religiosidad, a través de la regresión se explica el 42,6\% $[\mathrm{F}(6,1614)=$ $201,586, p \leq 0,01]$ de la varianza total. Se aprecia que en esta versión de la escala se excluyen nuevamente los ítems con tu salud $(B=0,034, p=$ $0,120)$ y con los grupos de los que formas parte $(B$ $=0,028, p=0,201)$.

Finalmente, en la regresión para la versión PWI + espiritualidad se explica el 41,4\% [F $(6,1614)$ $=191,937, p \leq 0,01]$ de la varianza. Nuevamente se excluyen los ítems con tu salud $(B=0,032, p=$ $0,156)$ y con los grupos de los que formas parte $(B$ $=0,027, p=0,233$ ).

De este modo, se aprecia la sistemática exclusión del modelo para cada una de las versiones del test de dos de los ítems originales. Asimismo, se refuerza el resultado del análisis factorial en orden a la baja en la varianza explicada a nivel nacional en relación con el reporte original del autor.

En las tablas 7, 8 y 9 se detallan los resultados para cada uno de los ámbitos en cada modelo propuesto, que incluyen los que demostraron niveles de significancia en las tres versiones del PWI. 
Tabla 7.

Contribución específica de ámbitos (PWI 7)

\begin{tabular}{|c|c|c|c|c|}
\hline Variables & $\begin{array}{c}\text { Correlación } \\
\text { parcial con } \\
\text { OLS }\end{array}$ & B & $\mathrm{t}$ & Sig. \\
\hline Seguridad sentida & 0,274 & 0,280 & 11,453 & 0,00 \\
\hline Nivel de vida & 0,208 & 0,193 & 8,563 & 0,00 \\
\hline $\begin{array}{l}\text { Seguridad a } \\
\text { futuro }\end{array}$ & 0,156 & 0,145 & 6,366 & 0,00 \\
\hline $\begin{array}{l}\text { Relación con las } \\
\text { personas }\end{array}$ & 0,148 & 0,132 & 6,001 & 0,00 \\
\hline Logros de vida & 0,112 & 0,112 & 4,547 & 0,00 \\
\hline
\end{tabular}

Nota: Significancia de correlaciones con $p \leq 0,01$. Fuente: autores.

Tabla 8.

Contribución específica de ámbitos

(PWI + religiosidad)

\begin{tabular}{lcccc}
\hline \multicolumn{1}{c}{ Variables } & $\begin{array}{c}\text { Correlación } \\
\text { parcial con } \\
\text { OLS }\end{array}$ & B & t & Sig. \\
\hline Seguridad sentida & 0,277 & 0,278 & 11,602 & 0,00 \\
Nivel de vida & 0,199 & 0,181 & 8,138 & 0,00 \\
Religión & 0,199 & 0,159 & 8,169 & 0,00 \\
$\begin{array}{l}\text { Seguridad a } \\
\text { futuro }\end{array}$ & 0,141 & 0,128 & 5,708 & 0,00 \\
Relación con las & 0,132 & 0,116 & 5,347 & 0,00 \\
personas & 0,105 & 0,103 & 4,262 & 0,00 \\
\hline Logros de vida & &
\end{tabular}

Nota: Significancia de correlaciones con $p \leq 0,01$.

Fuente: autores.

Tabla 9.

Contribución específica de ámbitos

(PWI + espiritualidad)

\begin{tabular}{lcccc}
\hline \multicolumn{1}{c}{ Variables } & $\begin{array}{c}\text { Correlación } \\
\text { parcial con } \\
\text { OLS }\end{array}$ & B & t & Sig. \\
\hline Seguridad sentida & 0,264 & 0,268 & 11,017 & 0,00 \\
Nivel de vida & 0,197 & 0,181 & 8,081 & 0,00 \\
Seguridad a & 0,148 & 0,136 & 6,026 & 0,00 \\
futuro & 0,140 & 0,118 & 5,681 & 0,00 \\
Espiritualidad & 0,128 & 0,114 & 5,172 & 0,00 \\
Relación con las & 0,099 & 0,098 & 3,985 & 0,00 \\
personas & Logros de vida & &
\end{tabular}

Nota: Significancia de correlaciones con $p \leq 0,01$.

Fuente: autores.
De acuerdo con lo contenido en las tres tablas precedentes, los ámbitos con lo seguro(a) que te sientes y con tu nivel de vida se sitúan, en las tres versiones, como los principales en la predicción respecto de OLS.

Cabe destacar que en la versión $P W I+$ religión, el ámbito con tu religión $[B=0,159, p \leq 001,95 \%$ IC $(0,075,0,122)]$ pasa a situarse en un tercer lugar en la explicación de OLS, a diferencia del ámbito con tu espiritualidad $[B=0,118, p \leq 001,95 \%$ IC $(0,061,0,125)]$ del $P W I+$ espiritualidad, que queda en un cuarto lugar en la explicación de la varianza para OLS.

Además es relevante indicar que en las tres versiones, el ámbito con las cosas que has conseguido en la vida presenta la menor contribución específica para OLS [PWI7: $B=0,112, p \leq 001,95 \%$ IC $(0,061,0,153)], P W I+$ religiosidad $[B=0,103, p \leq$ 001, 95\% IC $(0,053,0,144)]$ y $P W I+$ espiritualidad $[B=0,098, p \leq 001,95 \%$, IC $(0,047,0,139)]$.

\section{Conclusiones y discusiones}

Lo primero que se debe señalar es que los resultados de los coeficientes de confiabilidad (alfa de Cronbach) para las versiones del PWI 7 y $P W I+$ espiritualidad presentan valores similares $(0,805 \mathrm{y}$ 0,806 ), encontrándose dentro de los valores obtenidos por estudios internacionales (Chen y Davey, 2009; Lau et ál., 2005; Lau et ál., 2008; Davey, Chen y Lau, 2009; Nielsen, Smyth y Zhai, 2010; Nielsen, Paritski y Smyth, 2010; Rodríguez et ál., 2011; Webb, 2009). Ello da cuenta de un adecuado nivel de fiabilidad. La constatación de la baja comparativa del coeficiente de confiabilidad en la versión $P W I+$ religiosidad evidencia la dificultad en la inclusión específica de este campo de satisfacción, pese a mantenerse en rangos aceptables.

De modo complementario, los resultados de las correlaciones ítem-total, al excluirse los ítems religiosidad y espiritualidad, dan cuenta de valores que se encuentran en rangos similares a los arrojados por estudios a nivel internacional: de 0,33 a 0,69 para Hong Kong y de 0,21 a 0,66 para Australia (Lau et ál., 2005); de 0,30 a 0,56 
(Nielsen et ál., 2010a); de 0,35 a 0,50 (Nielsen et ál., 2010b); de 0,49 a 0,61 (Smith et ál., 2009) y de 0,47 a 0,61 (Chen y Davey, 2009). El decremento en los valores de las correlaciones en las versiones del PWI con inclusión de religiosidad y espiritualidad es concordante con los resultados que surgen del análisis de confiabilidad, en orden a dar cuenta de la menor asociación entre estos ámbitos en relación con los restantes. De este modo, se puede sostener que existe una adecuada consistencia interna de los instrumentos en estudio, siendo la versión PWI 7 la que evidencia una mayor confiabilidad en la aplicación.

Respecto a las correlaciones entre ámbitos, se aprecia que se encuentran en rangos similares a los obtenidos en estudios previos: de 0,06 a 0,59 (Nielsen et ál., 2010a); de 0,29 a 0,58 (Nielsen et ál., 2010b) y de 0,27 a 0,56 (Chen y Davey, 2009).

Según el análisis de componentes principales, la estructura monofactorial que emerge se encuentra en concordancia tanto con la perspectiva teórica a la base como con los hallazgos de estudios previos (Cummins et ál., 2003, 2004; Chen y Davey, 2009; Smyth et ál., 2010; Mellor et ál., 2009; Lau et ál., 2005). Asimismo, las cargas factoriales de los ámbitos "clásicos", excluyendo espiritualidad y religiosidad, se encuentran en rangos similares a los existentes en otros estudios: entre 0,77 y 0,66 (Tiliouine et ál., 2006) y entre 0,63 a 0,75 (Smyth et ál., 2009).

Los valores de varianza explicada que arroja el análisis factorial para las tres versiones de la escala son similares a los existentes en otros estudios: $43 \%$ (Smyth et ál., 2010) y 40\% a 48\% (Lau et ál., 2005). A su vez, son ligeramente mayores que los encontrados en otros estudios: $37,5 \%$ (Tiliouine et ál., 2006) y 38,3\% (Cummins et ál., 2003), dando cuenta así de una adecuada validez de constructo, similar al proyectado por los autores.

Respecto a la comparativa baja carga factorial del ámbito religiosidad (la menor de todas), se aprecia que se encuentra en concordancia con estudios previos (Casas et ál., 2009), lo que sustenta la reflexión en torno a la dificultad que dicho ámbito presenta al saturar en el mismo factor que los restantes ámbitos. De modo complementario, se aprecia que el ámbito espiritualidad se muestra relativamente cercano a los valores de saturación con el factor único.

A través del análisis de regresión lineal se aprecia que el rango de valores de $R^{2}$ para las tres versiones es relativamente inferior a lo obtenido en la mayoría de los estudios previos: 0,43 (Lau et ál., 2005), 0,44 (Nielsen et ál., 2010a), 0,46 (Smyth et ál., 2009), 0,47 (Chen y Davey, 2009), 0,50 (Nielsen et ál., 2010b), 0,52 (Cummins et ál., 2003) y 0,57 (Tiliouine et ál., 2006). Lo anterior da cuenta de la menor explicación de la satisfacción global por parte de los ámbitos definidos por el autor en un contexto social, económico y cultural distinto del que fueron diseñados.

Junto con lo anterior, se aprecia que la versión PWI + religiosidad es la que explica un mayor porcentaje de varianza, lo que en parte puede entenderse por la baja en la varianza explicada de la versión PWI 7, al contar de entrada con un ítem menos que las dos versiones alternativas. Complementariamente, se observa que la versión PWI + espiritualidad explica un menor porcentaje de varianza que la versión PWI + religiosidad (teniendo la misma cantidad de reactivos), a la vez que el valor $B$ del ámbito religiosidad es mayor que el valor B del ámbito espiritualidad. Estos resultados son aparentemente contradictorios en comparación con la menor carga factorial del ámbito religiosidad y su menor correlación ítem-test, con el consiguiente menor nivel de confiabilidad.

La preponderancia de los ámbitos seguridad y nivel de vida permiten establecer matices respecto a los estudios internacionales, en donde se posiciona primero el ámbito nivel de vida (Cummins y Lau, 2005; Tiliouine et ál., 2006; Nielsen et ál., 2010a; Nielsen et ál., 2010b) o seguridad en el futuro (Webb, 2009). Estos cambios en las preponderancias de los ámbitos de satisfacción, en conjunto con la menor varianza explicada desde la regresión, dan cuenta de los matices posibles en la aplicación del índice en realidades socioculturales distintas a las existentes en su construcción. 
Es interesante poder determinar la preponderancia de estos ámbitos en el caso de la muestra en estudio, junto con la existencia, en las tres versiones de la escala, de la exclusión sistemática de los ámbitos con tu salud y con los grupos de los que formas parte, desde los resultados de la regresión. Se entiende que estos análisis dan cuenta tanto de una específica etapa vital como, a su vez, de patrones culturales que relativizan y priorizan los ámbitos de satisfacción originalmente proyectados por el autor. Desde esta situación, la exclusión del ámbito con tu salud se puede explicar desde la escasa relevancia que tiene dicho ámbito para los adolescentes y jóvenes, en la medida que se encuentran en una etapa de la vida en la que, en términos generales, se goza de un buen estado físico y mental. Por otra parte, el no considerarse las relaciones con los grupos como un ámbito de satisfacción de relevancia puede dar cuenta de los procesos de cambio social en el contexto del proceso de modernización que se desarrolla en la sociedad chilena, con altas dosis de desvinculación y quiebre de los lazos comunitarios tradicionales.

Junto con lo anterior, es destacable que los ámbitos seguridad y nivel de vida sean los de mayor aporte a la explicación de la varianza, siendo, entonces, las condiciones materiales de vida y de seguridad las que predominan en la visión que tienen los adolescentes respecto a su satisfacción global. Estos resultados pueden explicarse desde la realidad sociocultural en la que se desenvuelven los sujetos de la muestra, que pertenecen a niveles socioeconómicos medio-bajo. De modo complementario, la política y el discurso social en Chile están fuertemente centrados en la noción de seguridad ciudadana.

Desde la menor varianza explicada para las tres versiones en estudio y la exclusión sistemática de dos de los ámbitos propuestos en el análisis de regresión, se plantea la relevancia de investigar acerca de la inclusión de ámbitos de satisfacción más acordes con los contextos socioculturales de países latinoamericanos, con la diversidad que supone dicha tarea. Lo anterior se conjuga con la relevancia política y social de abordar la construcción de indicadores de infancia que den cuenta efectiva tanto del bienestar psicológico como de la calidad de vida de la población infanto-juvenil, tanto en Chile como a nivel regional, dando pie a una mayor claridad en la aplicación y evaluación de las políticas públicas y de los programas hacia la infancia.

En síntesis, se aprecia que las tres versiones del PWI coinciden en la exclusión de los mismos ámbitos de satisfacción, a la vez que ponderan como prioritarios los mismos ámbitos (seguridad y nivel de vida). Asimismo, se aprecia que las versiones PWI 7 y PWI + espiritualidad presentan niveles de confiabilidad adecuados, lo que desciende en el PWI + religiosidad. Esta situación se complementa en el análisis de componentes principales, desde el cual se aprecia la menor carga factorial del ámbito religiosidad. Por lo anterior, consideramos que la versión PWI + espiritualidad es la más adecuada para la evaluación del bienestar subjetivo en adolescentes chilenos, en la medida que aporta un nuevo ámbito de relevancia, manteniendo propiedades psicométricas similares a las del PWI 7.

\section{Referencias}

Aguado Quintero, L. F. (2008). Un índice de no consumo de alimentos en la niñez para Colombia. Revista Latinoamericana de Ciencias Sociales: Niñez y Juventud, 7(2), 735-760.

Ben-Arieh, A. (2008). The child indicators movement: past, present and future. Child Indicators Research, 1, 3-16.

Bradshaw, J. \& Lau, M. (2010). Child well-being in the Pacific Rim. Child Indicators Research, 3(3), 367-383.

Casas, F. (2010a). Indicadores sociales subjetivos y bienestar en la infancia y adolescencia. En Unicef (Ed.). Propuesta de un sistema de indicadores sobre bienestar infantil en España (pp. 34-51). España: Unicef. 
Casas, F. (2010b). El bienestar personal: su investigación en la infancia y la adolescencia. Encuentros en Psicología Social, 5(1), 85-101.

Casas, F., González, M., Figuer, C. \& Malo, S. (2009). Satisfaction with spirituality, satisfaction with religion and personal well-being among Spanish adolescents and young university students. Applied Research in Quality of Life, 4, 23-45.

Casas, F., Sarriera, J., Abs da Cruz, D., Coenders, G., Alfaro, J., Saforcada, E. \& Tonon, G. (2011a). Subjective indicators of personal well-being among adolescents. Performance and results for different scales in LatinLanguage speaking countries: a contribution to the international debate. Child Indicators Research, 5, 1-28.

Casas, F., Sarriera, J., Alfaro, J., González, M., Malo, S. et ál. (2011b). Testing the personal well-being index on 12-16 year-old adolescents in 3 different countries with 2 new items. Social Indicators Research, 105, 461-482.

Chen, Z. \& Davey, G. (2009). Subjective quality of life in Zhuhai City, South China: a public survey using the International Wellbeing Index. Social Indicators Research, 91, 243-258.

Conicyt - Fondecyt (2008). Bioética en investigación en ciencias sociales. Santiago: Ministerio de Educación.

Cummins, R., Eckersley, R., Pallant, J., Van Vugt, J. \& Misajon, R. (2003). Developing a national index of subjective wellbeing: the Australian Unity Wellbeing Index. Social Indicators Research, 64, 159-190.

Cummins, R. A. \& Lau, A. (2005). The International Wellbeing Group Manual. Personal Wellbeing Index. Melbourne: Australian Centre on Quality of Life, Deakin University. Available in http://www.deakin.edu.au/research/acqol/ instruments/wellbeing_index.htm
Cummins, R. A., Lau, A. L. \& Stokes, M. (2004). Healthrelated quality of life and subjective wellbeing: noncomplementary forms of outcome measurement. Expert Review in Pharmacoeconomics Outcomes Research, 4, 89-96.

Davey, G., Chen, Z. \& Lau, A. (2009). Peace in a thatched hut-that is happiness: subjective wellbeing among peasants in rural China. Journal of Happiness Studies, 10, 239-252.

Diener, E. \& Suh, E. (1997). Measuring quality of live: economic, social and subjective indicators. Social Indicators Research, 40, 89-216.

Gademann, A, M., Schonert-Reichl, K. A. \& Zumbo, B. D. (2010). Investigating validity evidence of the satisfaction with Life Scale Adapted for Children. Social Indicators Research, 96, 229-247.

Huebner, E. S. (2004). Research on assessment of life satisfaction of children and adolescents. Social Indicators Research, 66(1-2), 3-33.

Huebner, E. S. \& Gilman, R. (2002). An introduction to the Multidimensional Students' Life Satisfaction Scale. Social Indicators Research, $60,115-122$.

International Wellbeing Group (2006). Personal Wellbeing Index. Melbourne: Australian Centre on Quality of Life, Deakin University. Available in http://www.deakin.edu.au/research/acqol/instruments/wellbeing_index. htm

Lau, A., Cummins, R. \& McPherson, W. (2005). An investigation into the cross-cultural equivalence of the Personal Wellbeing Index. Social Indicator Research, 72, 403-430.

Lau, A., Chi, I., Cummins, R., Lee, T. M., Chou, L.K. \& Chung, L. W. (2008). The SARS (Severe Acute Respiratory Syndrome) pandemic in Hong Kong: effects on the subjective wellbeing of elderly and younger people. Aging \& Mental Health, 12(6), 746-760. 
Mellor, D., Stokes, M., Firth, L., Hayashi, Y. \& Cummins, R. (2009). Need for belonging, relationship satisfaction, loneliness and life satisfaction. Personality and Individual Differences, 45, 213-218.

Nielsen, I., Paritski, O. \& Smyth, R. (2010). Subjective well-being of Beijing taxi drivers. Journal of Happiness Studies, 11, 721-733.

Nielsen, I., Smyth, R. \& Zhai, Q. (2010). Subjective Well-Being of China's Off-Farm Migrants. Journal of Happiness Studies, 11, 315-333.

Pavot, W. \& Diener, E. (1993). Review of the satisfaction with Life Scale. Psychological Assessment, 5(2), 164-172.

Pollard, E. \& Lee, P. D. (2003). Child well-being: a systematic review of the literature. Social Indicators Research, 61(1), 59-78.

Rodríguez, C., Frades, B., Forjaz, M., Ayala, A., Martínez, P., Fernández, G. \& Rojo, F. (2011). Psychometric properties of the International Wellbeing Index in community-dwelling older adults. International Psychogeriatrics, 23(1), 161-169

Seligson, J. L., Huebner, E. S. \& Valois, R. F. (2003). Preliminary validation of the Brief Multidimensional Students' Life Satisfaction Scale (BMSLSS). Social Indicators Research, 61, 121-145.

Seligson, J. L., Huebner, E. S. \& Valois, R. F. (2005). An investigation of a Brief Life Satisfaction Scale with elementary school children. Social Indicators Research, 73, 355-374.
Siyez, D. M. \& Kaya, A. (2008). With Turkish children validity and reliability of the Brief Multidimensional Students' Life Satisfaction Scale. Journal of Psychoeducational Assessment, 26(2), 139-147.

Smyth, R., Nielsen, I. \& Zhai, Q. (2010). Personal well-being in urban China. Social Indicators Research, 95, 231-251.

Tiliouine, H., Cummins, R. \& Davern, M. (2006). Measuring wellbeing in developing countries: the case of Algeria. Social Indicators Research, 75, 1-30.

Tomyn, A. J. \& Cummins, R. A. (2011). The subjective well-being of high-school students: validating the Personal Wellbeing Index School Children. Social Indicators Research, 101, 405-418.

Veenhoven, R. (1994). El estudio de la satisfacción con la vida. Intervención Psicosocial, 3(9), 87116, 125-127.

Veenhoven, R. (2002). Why social policy needs subjective indicators. Journal of Happiness Studies, 58, 33-46.

Webb, D. (2009). Subjective wellbeing on the Tibetan Plateau: an exploratory investigation. Journal of Happiness Studies, 10, 753-768.

Yiengprugsawan, V., Seubsman, S., Khamman, S. \&. Lim, L. L. (2010). Personal Wellbeing Index in a national cohort of 87, 134 Thai Adults. Social Indicators Research, 98, 201-215. 